\title{
Positive cultures and clinical outcomes in septic patients: be aware of the influence from patient selection and the in-hospital confounders
}

\author{
Romain Jouffroy ${ }^{*}$ (D) and Benoît Vivien \\ This comment refers to the article available at https://doi.org/10.1186/s13054-019-2431-8.
}

To the Editor:

We read with great interest the paper published by Nannan Panday et al. [1], who reported that, first, culturepositive sepsis patients have a higher mortality rate than those with culture-negative and, second, culture-positive sepsis patients more often have multiple organ systems affected during the sepsis episode. Firstly, we compliment the authors for this very interesting study.

Nevertheless, to our opinion, some methodological issues deserve their attention. Firstly, the database used for the analysis comes from another study (PHANTASi trial) [2], in which, eligible patients were those with a suspected of (severe) sepsis and septic shock [2]. Thus, we cannot be certain that all included patients have sepsis rather than an alternative diagnosis. The authors do not report how many patients were septic or not; we cannot rule out that patient selection could be the biggest issue directly affecting the study results [1]. Indeed, the blood culture results may be different if included patients were septic or not. This may also partly contribute to the negativity of the PHANTASi trial [2].

Secondly, the variables included in the multivariate analysis (age, group allocation, hospital location, source of infection, antibiotics at home and total amount of blood cultures drawn) do not consider the potential cofounders of the inhospital phase. Mortality is strongly affected by the inintensive care unit and in-hospital length of stay due to their potential complications [3]. For example, during the hospital stage, patients, especially elderly patients, may be affected by a limitation of care and/or nosocomial infection. Beyond these 2 methodological issues, we should keep in mind that, apart from source control, sepsis mortality is not only affected by antibiotherapy, but also by a bundle of care among which hemodynamic optimization plays an important role $[4,5]$.

Nevertheless, we fully agree that, contrary to septic shock, early identification of sepsis, especially those at risk of unfavourable evolution, is particularly difficult in the prehospital setting where the diagnosis is based on a list of non-specific physiological variables. The additional use of biomarkers, blood lactate measurement to assess severity and procalcitonin to confirm the bacterial origin of sepsis may be useful in order to define which patients should benefit from early prehospital antibiotic administration. Such a strategy enhancing better patients' selection could help the physician in the decision-making process and predict the real impact of early antibiotherapy.

\section{Authors' response}

\section{Rishi S. Nannan Panday, Eline M. J. Lammers and Prabath W. B. Nanayakkara}

Dear editor,

We've read with interest the letter to the editor by Jouffroy et al. We would like to thank the authors for

\footnotetext{
* Correspondence: romain.jouffroy@aphp.fr

SAMU de Paris, Service d'Anesthésie Réanimation, Hôpital Universitaire Necker - Enfants Malades, Assistance Publique - Hôpitaux de Paris, and Université Paris Descartes - Paris 5, Paris, France
}

their valuable input and hereby our reaction to their comments.

Firstly, the authors state that perhaps not all patients included in our study had sepsis, but they may have an alternative diagnosis. As described in our study, all patients met SEPSIS- 2 criteria at inclusion and retrospective chart analysis of all charts by a panel of experts 
(consisting of two acute physicians and one infectious disease specialist) was performed in order to exclude patients with an alternative diagnosis. This resulted in 1\% of patients in the intervention group and $2 \%$ in the control group with a different diagnosis. Although these patients were included in both the PHANTASi trial and our (sub) study, we believe that such small numbers will not have had an effect on the outcomes [1, 2].

Secondly, Jouffroy et al. raise the point that potential in-hospital confounders, such as length of stay and nosocomial infections, should be part of the multivariate regression model used in our study. However, we believe hospital length of stay may be part of the causal pathway, i.e. patients with positive cultures have a higher sepsis severity, are more critically ill and therefore have to stay in hospital longer. As described by Jager et al., including covariates that are to be part of the causal pathway of the outcome in a model may lead to overadjustment [6]. Indeed, nosocomial infections are common in sepsis patients, although studies report a low attributable risk of mortality caused by nosocomial infections [7]. We agree that superinfections should be considered as a covariate in future studies.

Apart from source control and antibiotic therapy, outcomes in septic patients are certainly effected by supportive care; however, hemodynamic optimization would mainly play a large role in septic shock patients, which were only approximately $4 \%$ of patients in our study cohort.

We agree with Jouffroy et al. that use of additional biomarkers, such as pro-calcitonin and lactate, in the (pre) hospital setting would guide emergency medical service personnel and clinicians to make a bacterial origin of infection more or less plausible. Currently, the level of the pro-calcitonin is not routinely measured in sepsis patients in the Netherlands, but this should be considered in the future. However, the role of procalcitonin in the emergency department setting is still a matter of debate.
Received: 2 August 2019 Accepted: 6 September 2019

Published online: 29 October 2019

\section{References}

1. Nannan Panday RS, Lammers EMJ, Alam N, Nanayakkara PWB. An overview of positive cultures and clinical outcomes in septic patients: a sub-analysis of the Prehospital Antibiotics Against Sepsis (PHANTASi) trial. Crit Care. 2019;23(1):182.

2. Alam N, Oskam E, Stassen PM, et al. Prehospital antibiotics in the ambulance for sepsis: a multicentre, open label, randomised trial. Lancet Respir Med. 2018;6(1):40-50.

3. Rannikko J, Syrjänen J, Seiskari T, Aittoniemi J, Huttunen R. Sepsis-related mortality in 497 cases with blood culture-positive sepsis in an emergency department. Int J Infect Dis. 2017;58:52-7.

4. Leisman DE, Doerfler ME, Ward MF, Masick KD, Wie BJ, Gribben JL, et al. Survival benefit and cost savings from compliance with a simplified 3-hour sepsis bundle in a series of prospective, multisite, observational cohorts. Crit Care Med. 2017:45:395-406.

5. Seymour $\mathrm{CW}$, Gesten F, Prescott $\mathrm{HC}$, et al. Time to treatment and mortality during mandated emergency care for sepsis. N Engl J Med. 2017;376:2235-44.

6. Jager KJ, Zoccali C, Macleod A, Dekker FW. Confounding: what it is and how to deal with it. Kidney Int. 2008;73(3):256-60.

7. Denstaedt SJ, Singer BH, Standiford TJ. Sepsis and nosocomial infection: patient characteristics, mechanisms, and modulation. Front Immunol. 2018;9:2446.

\section{Publisher's Note}

Springer Nature remains neutral with regard to jurisdictional claims in published maps and institutional affiliations.

\section{Acknowledgements}

None.

Authors' contributions

Both authors read and approved the final manuscript.

\section{Funding}

None.

Availability of data and materials

Not applicable.

Ethics approval and consent to participate

Not applicable.

Consent for publication

Not applicable.

Competing interests

The authors declare that they have no competing interests. 